\title{
Miniaturized Fluorimetric Method for Quantification of Zinc in Dry Dog Food
}

\author{
Rute C. Martins, ${ }^{1}$ Ana M. Pereira $\left(D,{ }^{2}\right.$ Elisabete Matos, ${ }^{3}$ Luisa Barreiros, ${ }^{1}$ \\ António J. M. Fonseca, ${ }^{2}$ Ana R. J. Cabrita, ${ }^{2}$ and Marcela A. Segundo $\mathbb{D}^{1}$ \\ ${ }^{1}$ LAQV, REQUIMTE, Departamento de Ciências Químicas, Faculdade de Farmácia, Universidade do Porto, \\ Rua Jorge Viterbo Ferreira no 228, Porto 4050-313, Portugal \\ ${ }^{2}$ LAQV, REQUIMTE, Instituto de Ciências Biomédicas de Abel Salazar (ICBAS), Universidade do Porto, \\ Rua de Jorge Viterbo Ferreira no 228, Porto 4050-313, Portugal \\ ${ }^{3}$ SORGAL, Sociedade de Óleos e Rações S.A., Estrada Nacional 109 Lugar da Pardala, São João 3880-728, Ovar, Portugal \\ Correspondence should be addressed to Marcela A. Segundo; msegundo@ff.up.pt
}

Received 7 April 2020; Accepted 12 August 2020; Published 4 September 2020

Academic Editor: María José Trujillo Rodríguez

Copyright () 2020 Rute C. Martins et al. This is an open access article distributed under the Creative Commons Attribution License, which permits unrestricted use, distribution, and reproduction in any medium, provided the original work is properly cited.

\begin{abstract}
Zinc is an essential trace element for animals in several biological processes, particularly in energy production, and it is acquired from food ingestion. In this context, a microplate-based fluorimetric assay was developed for simple, fast, and low-cost determination of zinc in pet food using 2,2'-((4-(2,7-difluoro-3,6-dihydroxy-4aH-xanthen-9-yl)-3-methoxyphenyl)azanediyl) diacetic acid (FluoZin-1) as fluorescent probe. Several aspects were studied, namely, the stability of the fluorescent product over time, the FluoZin- 1 concentration, and the $\mathrm{pH}$ of reaction media. The developed methodology provided a limit of detection of $1 \mu \mathrm{g}$ $\mathrm{L}^{-1}$ in sample acid digests, with a working range of 10 to $200 \mu \mathrm{g} \mathrm{L}^{-1}$, corresponding to $100-2000 \mathrm{mg}$ of $\mathrm{Zn}$ per $\mathrm{kg}$ of dry dog food samples. Intraday repeatability and interday repeatability were assessed, with relative standard deviation values $<3.4 \%(100 \mu \mathrm{g}$ $\left.\mathrm{L}^{-1}\right)$ and $<11.7 \%\left(10 \mu \mathrm{g} \mathrm{L}^{-1}\right)$. Sample analysis indicated that the proposed fluorimetric assay provided results consistent with ICPMS analysis. These results demonstrated that the developed assay can be used for rapid determination of zinc in dry dog food.
\end{abstract}

\section{Introduction}

Zinc is an important element in the feeding of living beings, as it intervenes in the metabolism of proteins and nucleic acids, as well as in the appropriate functioning of biological systems. It exists in the whole body, as zinc(II) or $\mathrm{Zn}^{2+}$, mainly as an intracellular constituent, and it is present in most tissues at relatively low concentrations [1]. Concerning the existence in dogs, total body $\mathrm{Zn}$ content of puppies and young adult dogs is on average 23.1 and $9.5 \mathrm{mg} \cdot \mathrm{kg}^{-1} \mathrm{BW}$ (body weight), respectively, and it is acquired through the ingested food [2].

The absorption of dietary zinc might be affected by other dietary constituents, which alters its bioavailability from food. The absorption of zinc can be disturbed by compounds from the vegetable ingredients present in dog food, among which phytate assumes particular importance. In fact, dietary phytate reduces the absorption of zinc, and this effect is aggravated by high concentrations of edible calcium. Furthermore, the zinc deficiency is commonly correlated with growth retardation in young animals [3]. Zinc is an almost nontoxic substance, but it can sometimes become toxic when it interacts with other nutrients in the animal's body. A few cases of inadvertent overconsumption of zinc by dogs, namely, by puppies, have been reported, and the clinical signs detected included acute gastroenteritis and anemia [3]. For zinc in particular, the minimum recommended level in complete food for adult dogs is 7.20 to $8.34 \mathrm{mg}$ per $100 \mathrm{~g}$ of dry matter, with a higher level for puppies $(10.00 \mathrm{mg}$ per $100 \mathrm{~g}$ of dry matter) [4]. There is also a maximum legal limit established in the $\mathrm{EU}$, corresponding to $22.7 \mathrm{mg}$ per $100 \mathrm{~g}$ of dry matter. [5]. 


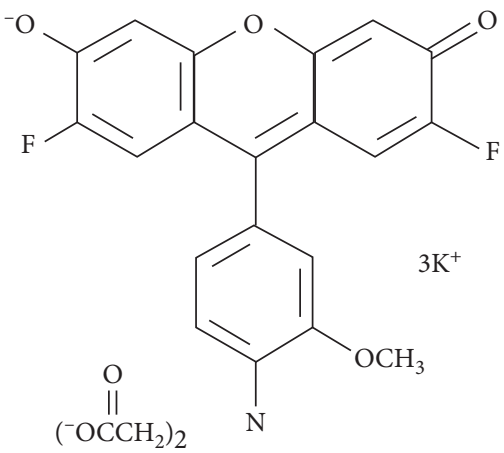

Figure 1: Structure of 2,2'-((4-(2, 7-difluoro-3,6-dihydroxy-4aHxanthen-9-yl)-3-methoxyphenyl)azanediyl)diacetic acid (FluoZin-1).

The need for quality control, particularly during formulation of commercial dry dog food, requires fast and simple methods for zinc determination. To date, various methods have been proposed to determine zinc, namely, conventional methods based on atomic features, including inductively coupled plasma mass spectrometry (ICP-MS) [6, 7], atomic emission spectrometry (ICP-AES) [8], atomic absorption spectrophotometry (AAS) [9], and energy dispersive X-ray fluorescence (ED-XRF) [10]. Nevertheless, these methods require trained personnel and high-cost/high maintenance equipment. Thus, there are some rapid and low-cost methods for zinc determination, based on fluorimetric [11] or colorimetric assays [12,13] and on nearinfrared reflectance spectroscopy [14].

Several fluorimetric probes are available for assessment of zinc [15], particularly tailored for zinc assay in living cells using microscopy. FluoZin-1 (2,2'-((4-(2,7-difluoro-3,6dihydroxy-4aH-xanthen-9-yl)-3-methoxyphenyl)azanediyl) diacetic acid), for instance, has been initially proposed for intracellular $\mathrm{Zn}^{2+}$ imaging and quantitation in the low hundred nanomolar concentration range, as it presents a large dynamic range. Other applications of this probe include the determination of stability constants of Cd(II) and $\mathrm{Zn}$ (II) complexes with thiols [16], the estimation of the apparent dissociation constant of bovine serum albumin for $\mathrm{Zn}^{2+}$ binding [17], and the evaluation of proton-dependent transport of $\mathrm{Zn}^{2+}$ mediated by integral synaptic vesicle protein SV31 in a proteoliposome model [18].

In this context, miniaturization of (bio)chemical reactions and analytical methods is a current trend, using either microchips $[19,20]$ or downscaling under microplate format $[21,22]$, fostering greener methodologies that originate less effluent, consume fewer reagents, and have a lower environmental footprint. Taking into account the need to develop a simple, fast, sensitive, and precise methodology for zinc determination to support pet food formulation using different sources of bioavailable zinc, the objective of this work is to implement a fluorimetric methodology based on FluoZin-1, aiming for the determination of zinc in pet food samples after acid digestion.

\section{Materials and Methods}

2.1. Chemicals and Solutions. All chemicals used in this work were of analytical reagent grade with no further purification and purchased from Sigma-Aldrich (St. Louis MO, USA) unless otherwise stated. Ultrapure water $(18.2 \mathrm{M} \Omega \mathrm{cm})$ used in all experiments was obtained from a Sartorius Arium ${ }^{\circledR}$ water purification system (Goettingen, Germany). Sample digestion was performed using high-purity $\mathrm{HNO}_{3}(\geq 69 \%$ (w/w), TraceSELECT ${ }^{\circledR}$ (Fluka, Seelze, Germany)), and $\mathrm{H}_{2} \mathrm{O}_{2}$ $\left(30 \%(\mathrm{v} / \mathrm{v})\right.$, TraceSELECT ${ }^{\circledR}$ Fluka) of p.a. grade. All plasticware used in the sample digestion and elemental analysis was immersed for, at least, $24 \mathrm{~h}$ in a $10 \%(\mathrm{v} / \mathrm{v}) \mathrm{HNO}_{3}$ solution to ensure decontamination and then rinsed with ultrapure water.

The fluorescent reagent FluoZin-1 $\left(2,2^{\prime}-((4-(2,7-\right.$ difluoro-3,6-dihydroxy-4aH-xanthen-9-yl)-3-methox-

yphenyl)azanediyl)diacetic acid, Figure 1), tripotassium salt, cell impermeant (catalog number F24180) was acquired from Invitrogen-Thermo Fisher Scientific (Massachusetts, USA). Stock standard solutions of FluoZin-1 were prepared by dissolving the solid in $1 \mathrm{~mL}$ dimethyl sulfoxide (DMSO) from Merck (Darmstadt, Germany), resulting in a $0.5 \mathrm{mg} \cdot \mathrm{mL}^{-1}$ probe solution that was stored at $-20^{\circ} \mathrm{C}$ (with fluorescence stability during six months). Intermediate solutions of $25.0,5.0,2.5$, and $1.25 \mu \mathrm{M}$ were prepared prior to use using either buffer or ultrapure water, depending on the experimental work performed.

The standard stock solution of $\mathrm{Zn}(\mathrm{II})\left(1.00 \mathrm{mg} \cdot \mathrm{mL}^{-1}\right)$ in $4 \%$ nitric acid was obtained by SCP Science (item 140-001301, $125 \mathrm{~mL}$, UN Code 3264, Baie-d'Urfe, Canada). Moreover, the stock solution of nitric acid $10 \mathrm{mM}$, used to prepare the zinc(II) standard working solutions, was prepared by diluting commercial nitric acid $70 \%\left(\mathrm{w} \mathrm{w}^{-1}\right)$.

Several buffer solutions were used, namely, acetic acid/ sodium acetate $(1000 \mathrm{mM}, \mathrm{pH} 3.9)$, potassium phosphate dibasic/potassium phosphate monobasic (1000 mM, pH 7.0), and sodium hydrogen carbonate/sodium carbonate $(1000 \mathrm{mM}, \mathrm{pH} 10.0)$. The working buffer solution (potassium phosphate dibasic/potassium phosphate monobasic, $\mathrm{pH} 7.0$ ) at $5 \mathrm{mM}$ was prepared by $1: 20$ dilution from a $100 \mathrm{mM}$ buffer solution.

\subsection{Fluorescence Measurements and Microplate Protocol.} Fluorescence measurements were carried out using a Cytation $3^{\circledR}$ microplate reader (Bio-Tek Instruments, Winooski, USA), controlled by the Gen5 (Bio-Tek Instruments) software. Solutions were placed in 96-well microplates, suitable for fluorescence measurements (black, opaque wells, ref. 10588885, Thermo Fisher Scientific, Massachusetts, USA). The excitation wavelength was set to $484 \mathrm{~nm}$ while the emission wavelength was $520 \mathrm{~nm}$.

The final volume of the reaction mixture was set to $260 \mu \mathrm{L}$ and the assay procedure comprised the sequential addition of $160 \mu \mathrm{L}$ of phosphate buffer solution $(5 \mathrm{mM}$, pH 7.0), $80 \mu \mathrm{L}$ of zinc(II) standard solution, and $20 \mu \mathrm{L}$ of FluoZin-1 solution to each well. For sample analysis, the protocol was adapted, comprising the addition of $160 \mu \mathrm{L}$ of water, $80 \mu \mathrm{L}$ of zinc(II) standard solution or diluted sample digest, and $20 \mu \mathrm{L}$ of FluoZin-1 prepared in phosphate buffer solution. Control experiments were performed by replacing each solution by their respective solvent. 
A conventional fluorimeter (Model FP-6500, JASCO, Easton, USA) was applied to evaluate the excitation and emission spectra in initial studies.

2.3. Analysis of Pet Food Samples. Dry dog food samples were acquired in local supermarkets. Each sample was digested in duplicate, after a digestion pretreatment procedure, where the samples were oven-dried at $65^{\circ} \mathrm{C}$ to constant weight and then ground in a $1 \mathrm{~mm}$ sieve mill. Ground samples ( $c a$. $500 \mathrm{mg}$ ) were solubilized by microwave-assisted acid digestion as described by Pereira et al. [7], using an MLS 1200 Mega high-performance microwave digestion unit (Milestone, Sorisole, Italy) equipped with an HPR-1000/10 S rotor. After weighing the sample using a plastic spatula, $3 \mathrm{~mL}$ of $\mathrm{HNO}_{3}$ and $1 \mathrm{~mL}$ of $\mathrm{H}_{2} \mathrm{O}_{2}$ were added to each polytetrafluoroethylene digestion vessel. The samples were subsequently submitted to a microwave heating program of $250 \mathrm{~W}$ for $1 \mathrm{~min}, 0 \mathrm{~W}$ for $1 \mathrm{~min}, 250 \mathrm{~W}$ for $5 \mathrm{~min}, 400 \mathrm{~W}$ for $5 \mathrm{~min}$, and, finally, $650 \mathrm{~W}$ for $5 \mathrm{~min}$. The vessels were then allowed to cool to room temperature. Thereafter, the content was transferred to $25 \mathrm{~mL}$ polypropylene volumetric flasks and water was added to bring up to total volume. This digest was analyzed by the developed fluorimetric assay. A blank constituted by $500 \mu \mathrm{L}$ of water was included in each digestion run.

For comparison purposes, zinc present in sample digests was also determined by inductively coupled plasma mass spectrometry (ICP-MS) using an iCAP $\mathrm{Q}^{\mathrm{TM}}$ (Thermo Fisher Scientific, Schwerte, Germany) instrument, equipped with a MicroMist $^{\mathrm{TM}}$ nebulizer, a Peltier cooled cyclonic spray chamber, a standard quartz torch, and nickel skimmer and sampling cones. High-purity (99.9997\%) Ar (Gasin II, Leça da Palmeira, Portugal) was used as the nebulizer and plasma gas. The ICP-MS operated under the following conditions: RF power $1550 \mathrm{~W}$; auxiliary Ar flow rate $0.80 \mathrm{~L} \mathrm{~min}^{-1}$; nebulizer flow rate $1.08 \mathrm{~L} \mathrm{~min}^{-1}$, and plasma flow rate $14 \mathrm{~L} \mathrm{~min}^{-1}$. Zinc was determined as ${ }^{6} \mathrm{Zn}^{\mathrm{n}}$ isotopes as described in detail elsewhere [23].

\section{Results and Discussion}

3.1. Implementation of Fluorescence Reaction under Microplate Format. Initially, the excitation and emission spectrum of FluoZin-1 probe was evaluated using a conventional spectrofluorimeter. An increase of fluorescence intensity was observed in the presence of zinc(II), compared to the probe self-fluorescence, in the emission wavelength range 510 to $530 \mathrm{~nm}$. For the excitation spectra, fluorescence intensity increased for higher wavelengths, showing a plateau at $460-470 \mathrm{~nm}$, and further increased up to $490 \mathrm{~nm}$. In fact, the wavelengths corresponding to the maximum fluorescence intensity were 495 and $517 \mathrm{~nm}$ for excitation and emission, respectively.

To transpose the detection of $\mathrm{Zn}$ (II)-FluoZin-1 to the current microplate equipment, a difference of at least $30 \mathrm{~nm}$ between excitation and emission wavelength is recommended. Therefore, the excitation wavelength was set to $484 \mathrm{~nm}$ while the emission wavelength was $520 \mathrm{~nm}$, corresponding to the maximum emission observed in the microplate reader (Figure 2(a)).

The implementation of fluorimetric assay under microplate format required a careful choice of employed volumes. First, the volume of buffer solution was fixed at $160 \mu \mathrm{L}$, accounting for at least $50 \%$ of the total volume and ensuring suitable $\mathrm{pH}$ adjustment. For standard/sample, the volume chosen was $80 \mu \mathrm{L}$, as the expected values in pet food acid digest would be in the $\mu \mathrm{g}$ per $\mathrm{L}$ range, providing an adequate sensitivity. Finally, considering the cost of the fluorescence probe, a lower volume was chosen $(20 \mu \mathrm{L})$, still high enough to be handled by conventional micropipettes.

Next, the effect of the FluoZin-1 concentration was evaluated. For the three tested concentrations $(1.25,2.5$, and $5.0 \mu \mathrm{M})$, there was an increase in fluorescence intensity with FluoZin-1 concentration, causing also an increase of fluorescence background $(277 \pm 9,546 \pm 6$, and $1186 \pm 44)$ when increasing FluoZin-1 concentration. An increase in fluorescence intensity proportional to the amount of zinc(II) present in the reaction medium was also observed (tested $\mathrm{Zn}(\mathrm{II})$ concentrations of $\left.50-1000 \mu \mathrm{g} \mathrm{L}^{-1}\right)$. The sensitivity was higher for increasing concentrations of FluoZin-1, with slope values of $5.46 \pm 0.29,11.4 \pm 0.5$, and $22.6 \pm 0.9 \mathrm{~L} \cdot \mu \mathrm{g}^{-1}$, respectively. Further experiments were performed using $2.5 \mu \mathrm{M}$ as a compromise between sensitivity and analysis cost.

Using these conditions, the fluorescence stability over time was evaluated (Figure 2(b)). For all zinc(II) concentrations, the fluorescence was stable during $4 \mathrm{~h}$, showing values corresponding to 95.6 to $98.3 \%$ in relation to the initial fluorescence intensity. Moreover, the fluorescence from FluoZin-1 in the absence of $\mathrm{Zn}$ (II) was also constant (Figure 2(b), A), showing no sign of photobleaching.

Subsequently, the influence of $\mathrm{pH}$ of reaction media on fluorescence value was studied (Figure 3). FluoZin-1 probe was not effective for $\mathrm{Zn}$ (II) determination at acidic $\mathrm{pH}$ as the attained fluorescence was constant or even decreased in the presence of increasing zinc(II) concentrations. This can be explained by the fact that the carboxylic groups are ionized for $\mathrm{pH}$ values $>4$, but the hydroxyl group on the unsaturated ring system presents a $\mathrm{pKa}$ value of 5.4, which can explain the deactivation of fluorescence observed for $\mathrm{pH}$ values $<6.0$. For reaction media at $\mathrm{pH} 7.0$ and $\mathrm{pH} 8.0$, an adequate response concerning a direct relation between fluorescence and $\mathrm{Zn}$ (II) concentration was observed because both carboxylic groups and the hydroxyl group are ionized. In relation to reaction media at $\mathrm{pH} 9.0$, a proportional response was also observed, but a decrease in the fluorescence intensity occurred for $\mathrm{Zn}\left(\mathrm{II}\right.$ ) concentrations above $500 \mu \mathrm{g} \mathrm{L} \mathrm{L}^{-1}$ (data not shown). Therefore, further experiments were performed at $\mathrm{pH} 7.0$.

3.2. Analytical Features. Calibration curves were linear from 10 to $200 \mu \mathrm{g} \mathrm{L}^{-1}$, with a typical calibration curve of $y=24.5$ $( \pm 0.2) x+1027 \pm 21, R=0.9993$, where $y$ is the fluorescence intensity and $x$ is the concentration of $\mathrm{Zn}$ (II) expressed in $\mu \mathrm{g}$ 


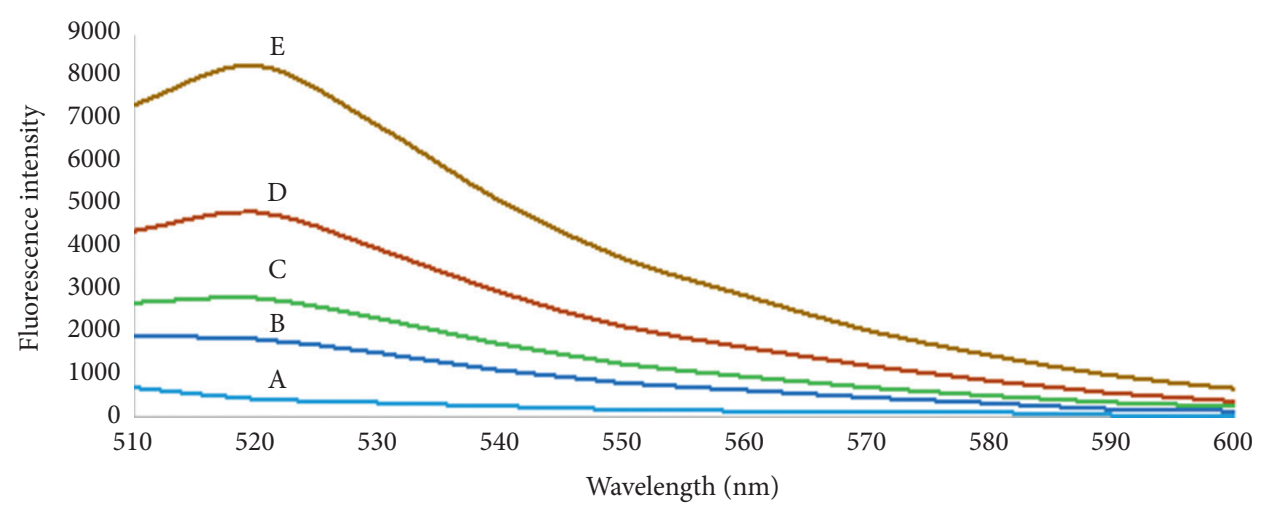

(a)

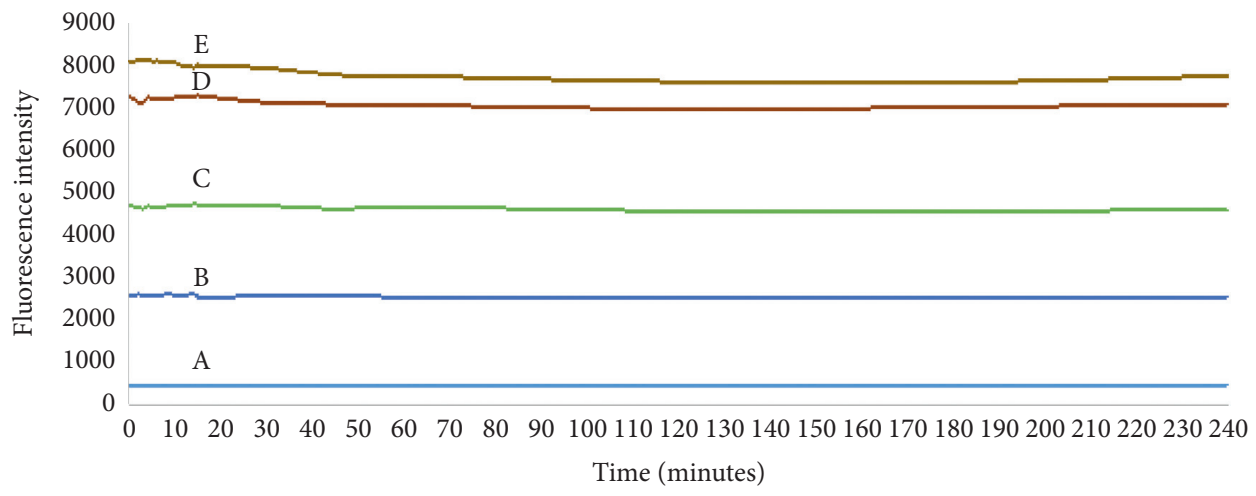

(b)

FIgURE 2: (a) FluoZin-1 probe $(1.25 \mu \mathrm{M})$ emission spectra $(\lambda$ exc $=495 \mathrm{~nm})$ with different concentrations of zinc(II). (b) Fluorescence intensity along time, using FluoZin-1 probe $(1.25 \mu \mathrm{M})$ and different concentrations of zinc(II) $\left(\mu \mathrm{g} \mathrm{L} \mathrm{L}^{-1}\right)$ : (A) 0, (B) 100 , (C) 200, (D) 500 , (E) 1000 .

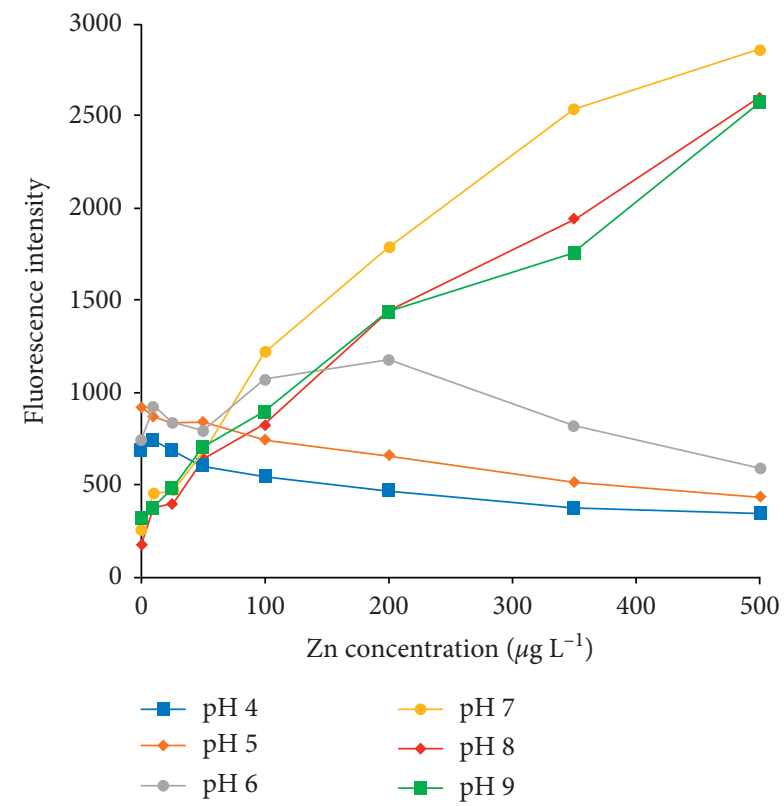

FIGURE 3: Fluorescence intensity of FluoZin-1 probe at different $\mathrm{pH}$ values (phosphate buffer).

$\mathrm{L}^{-1}$. This working range corresponds to $100-2000 \mathrm{mg}$ of $\mathrm{Zn}$ per $\mathrm{kg}^{-1}$ of dry dog food samples considering the use of $0.5 \mathrm{~g}$ of sample and the digestion procedure described above. The limits of detection and quantification were 1 and $9 \mu \mathrm{g} \mathrm{L}^{-1}$, respectively, estimated by interpolation of the blank signal $(n=9)$ plus 3 or 10 times its standard deviation [24]. Accuracy and repeatability data are presented in Table 1. Accuracy was evaluated as the percentage ratio between the calculated and nominal concentrations, with acceptable values between 96.1 and $100.2 \%$. Intraday and interday precision values were expressed as relative standard deviations (RSD\%) [24], with values $<3.4 \%$ for $\mathrm{Zn}$ (II) concentration $>100 \mu \mathrm{g} \mathrm{L}^{-1}$ and $<11.7 \%$ for $\mathrm{Zn}(\mathrm{II})$ concentration of $10 \mu \mathrm{g} \mathrm{L}^{-1}$.

3.3. Analysis of Pet Food Samples. After establishing the reaction conditions for $\mathrm{Zn}$ (II) determination using FluoZin1 probe, samples were analyzed after microwave-assisted acid digestion. Initially, a digested sample was diluted $1: 1$ in water and processed by the established protocol. The fluorescence intensity was similar to that presented in the absence of zinc and remained the same after fortification with $\mathrm{Zn}$ (II) at $500 \mu \mathrm{g} \mathrm{L}^{-1}$. In order to investigate if this effect was due to sample $\mathrm{pH}$, digested samples and a digested blank were analyzed after dilution, with an equivalent content in nitric acid of $1.5,3.0$, and $6.0 \mathrm{mM}$. The two most acidic solutions did not provide different results among the samples and blank while, for the less acidic solutions $\left(1.5 \mathrm{mM}\right.$ of $\left.\mathrm{HNO}_{3}\right)$, the samples presented higher 
TABLE 1: Accuracy and precision using the conditions established for the analysis of $\mathrm{Zn}$ (II) in pet food.

\begin{tabular}{|c|c|c|c|c|}
\hline \multirow[b]{2}{*}[\mathrm{Zn}(\mathrm{II})]{$\left(\mu \mathrm{g} \mathrm{L}^{-1}\right)$} & \multicolumn{2}{|l|}{ Intraday } & \multicolumn{2}{|l|}{ Interday } \\
\hline & Back-calculated concentration (\%) & RSD (\%) & Back-calculated concentration (\%) & RSD (\%) \\
\hline 10 & 100.2 & 11.7 & 107.1 & 9.2 \\
\hline 100 & 96.1 & 2.0 & 95.0 & 1.7 \\
\hline 200 & 98.7 & 1.0 & 96.4 & 3.4 \\
\hline
\end{tabular}

TABle 2: $\mathrm{Zn}$ (II) amount in pet food samples expressed as $\mathrm{mg}$ of $\mathrm{Zn}$ per kg of dry food.

\begin{tabular}{lcccc}
\hline Sample & $\begin{array}{c}\text { Proposed } \\
\text { method }\end{array}$ & $\begin{array}{c}\text { ICP-MS } \\
\text { method }\end{array}$ & $\begin{array}{c}\text { Absolute } \\
\text { deviation }\end{array}$ & $\begin{array}{c}\mathrm{Rd} \\
(\%)\end{array}$ \\
\hline $\mathrm{A}$ & $393 \pm 14$ & $357 \pm 15$ & 36 & 10.2 \\
$\mathrm{~B}$ & $472 \pm 13$ & $421 \pm 30$ & 51 & 12.1 \\
$\mathrm{C}$ & $336 \pm 10$ & $342 \pm 32$ & -6 & -1.8 \\
$\mathrm{D}$ & $433 \pm 25$ & $378 \pm 22$ & 55 & 14.6 \\
$\mathrm{E}$ & $279 \pm 20$ & $286 \pm 10$ & -7 & -2.6 \\
$\mathrm{~F}$ & $297 \pm 43$ & $256 \pm 1$ & 41 & 15.8 \\
$\mathrm{G}$ & $225 \pm 9$ & $243 \pm 3$ & -18 & -7.4 \\
$\mathrm{H}$ & $228 \pm 19$ & $243 \pm 2$ & -15 & -6.2 \\
\hline
\end{tabular}

fluorescence when compared to the blank and this difference was increased upon fortification with $\mathrm{Zn}(\mathrm{II})$ at $200 \mu \mathrm{g} \mathrm{\textrm {L } ^ { - 1 }}$. Sample $\mathrm{pH}$ was an issue and the final procedure for sample analysis included a step for $\mathrm{pH}$ adjustment to 3.6 (and dilution $1: 200)$ before analysis. Therefore, the protocol was adapted and $160 \mu \mathrm{L}$ of water was used instead of $160 \mu \mathrm{L}$ of buffer solution. As the FluoZin-1 probe solution was prepared in phosphate buffer $\mathrm{pH} 7.0$, it assured that the reaction medium was at a $\mathrm{pH}$ value adequate for fluorescence measurements.

Sample digests were analyzed by the proposed method and by ICP-MS, providing the values reported in Table 2, expressed as $\mathrm{mg}$ of $\mathrm{Zn}$ per $\mathrm{kg}$ of dry food. Good agreement was obtained between the two methodologies, with relative deviations between -7.4 and $15.8 \%$. A paired $t$-test was performed on the data obtained for these samples and a $t$ value of 0.417 was calculated, which was compared to the tabulated $t$ (2.144, $P=0.05, \mathrm{df}=14$ ), indicating no significant difference for the mean concentration obtained by the two methods [25]. The estimated cost of analysis per microplate was $2.70 €$, accounting for a value of $0.028 €$ per determination.

Compared to a recent work using a microplate colorimetric assay [13], the strategy proposed here is more straightforward as it does not require the inclusion of ion masking reagents or the application of partitioning correction calculations. Compared to the fast method proposed by Goi et al. [14], our approach provides the value of the total $\mathrm{Zn}$ as a digestion process is performed before the fluorimetric reaction while the near-infrared reflectance spectroscopy-based method can only detect $\mathrm{Zn}$ associated with organic complexes, providing underestimated results for dog food supplemented with inorganic forms of $\mathrm{Zn}$. Finally, compared to other approaches that applied AAS [26] or ICPMS [7], the method proposed here allows the simultaneous determination of up to 96 samples, without requiring expensive equipment or application of the standard addition method.

\section{Conclusions}

A low-cost, environmentally friendly method for determination of zinc in dry dog food samples was developed, resorting to an innovative application of FluoZin-1. The proposed microplate format fostered the use of low amounts of reagents ( $<0.1 \mathrm{nmol}$ of fluorescent probe), also generating a small amount $(260 \mu \mathrm{L})$ of effluent per assay. Moreover, high-throughput analysis was implemented, allowing for the simultaneous reading of up to 96 samples simultaneously, without using high maintenance/high-cost equipment based on AAS or ICP-MS. The application of the proposed methodology to the fast evaluation of zinc content during pet food formulation is envisioned.

\section{Data Availability}

Data are available upon request to the corresponding author.

\section{Conflicts of Interest}

The authors declare no conflicts of interest.

\section{Acknowledgments}

The authors thank the financial support of the MinDog project, funded by Portugal 2020 program, through the European Regional Development Fund (FEDER) through the Competitiveness and Internationalization Operational Program (COMPETE) (nr. 017616 (POCI-01-0247-FEDER017616). This work also received financial support from PT national funds (FCT/MCTES, Fundação para a Ciência e a Tecnologia, and Ministério da Ciência, Tecnologia e Ensino Superior) through Grant UIDB/50006/2020. L. Barreiros thanks FCT for funding through program DL 57/2016Norma Transitória. A.M. Pereira also thanks FCT, the SANFEED Doctoral Program, Soja de Portugal, and Alltech for funding her Ph.D. scholarship (PD/BDE/114427/2016).

\section{References}

[1] K. Kaur, R. Gupta, S. A. Saraf, and S. K. Saraf, "Zinc: the metal of life," Comprehensive Reviews in Food Science and Food Safety, vol. 13, no. 4, pp. 358-376, 2014.

[2] National Research Council, Nutrient Requirements of Dogs and Cats, The National Academies Press, Washington, DC, USA, 2006.

[3] M. S. Hand, C. D. Thatcher, R. L. Remillard, P. Roudebush, and B. J. Novotny, Small Animal Clinical Nutrition, Mark Morris Associates, Asheville, NC, USA, 5th edition, 2010. 
[4] FEDIAF, The European Pet Food Industry Federation, Nutritional Guidelines for Complete and Complementary Pet Food for Cats and Dogs, FEDIAF, Brussels, Belgium, 2019.

[5] European Comission, Commission Implementing Regulation (EU) 2016/1095 of 6 July 2016.

[6] T. King and R. Sheridan, "Determination of 27 elements in animal feed by inductively coupled plasma-mass spectrometry," Journal of AOAC International, vol. 102, no. 2, pp. 434-444, 2019.

[7] A. M. Pereira, E. Pinto, E. Matos et al., "Mineral composition of dry dog foods: impact on nutrition and potential toxicity," Journal of Agricultural and Food Chemistry, vol. 66, no. 29, pp. 7822-7830, 2018.

[8] H.-t. Kim, J. Loftus, J. W. Gagne, M. A. Rutzke, R. P. Glahn, and J. J. Wakshlag, "Evaluation of selected ultra-trace minerals in commercially available dry dog foods," Veterinary Medicine: Research and Reports, vol. 9, pp. 43-51, 2018.

[9] A. Duran, M. Tuzen, and M. Soylak, "Trace element concentrations of some pet foods commercially available in Turkey," Food and Chemical Toxicology, vol. 48, no. 10, pp. 2833-2837, 2010.

[10] L. Perring, M. Nicolas, D. Andrey et al., "Development and validation of an ED-XRF method for the fast quantification of mineral elements in dry pet food samples," Food Analytical Methods, vol. 10, no. 5, pp. 1469-1478, 2017.

[11] Z. Amouzegar, A. Afkhami, and T. Madrakian, "ZnS quantum dots surface-loaded with zinc(ii) ions as a viable fluorescent probe for glutathione," Microchimica Acta, vol. 186, no. 3, 2019.

[12] F. Li, Y. Hu, Z. Li, J. Liu, L. Guo, and J. He, “Three-dimensional microfluidic paper-based device for multiplexed colorimetric detection of six metal ions combined with use of a smartphone," Analytical and Bioanalytical Chemistry, vol. 411, no. 24, pp. 6497-6508, 2019.

[13] J. Wang, Y. Niu, C. Zhang, and Y. Chen, "A micro-plate colorimetric assay for rapid determination of trace zinc in animal feed, pet food and drinking water by ion masking and statistical partitioning correction," Food Chemistry, vol. 245, pp. 337-345, 2018.

[14] A. Goi, C. L. Manuelian, S. Curro, and M. De Marchi, "Prediction of mineral composition in commercial extruded dry dog food by near-infrared reflectance spectroscopy," Animals, vol. 9, no. 9, 2019.

[15] K. R. Gee, Z.-L. Zhou, D. Ton-That, S. L. Sensi, and J. H. Weiss, "Measuring zinc in living cells," Cell Calcium, vol. 31, no. 5, pp. 245-251, 2002.

[16] M. J. Walsh and B. A. Ahner, "Determination of stability constants of $\mathrm{Cu}(\mathrm{I}), \mathrm{Cd}(\mathrm{II})$ \& $\mathrm{Zn}(\mathrm{II})$ complexes with thiols using fluorescent probes," Journal of Inorganic Biochemistry, vol. 128, pp. 112-123, 2013.

[17] Y. Zheng, L. Lin, W. Hang, X. Yan, and B. L. Marrone, "Analysis of beryllium to biomolecule binding using a metal specific fluorescent probe and competitive assay," Talanta, vol. 85, no. 1, pp. 638-643, 2011.

[18] L. Waberer, E. Henrich, O. Peetz et al., "The synaptic vesicle protein SV31 assembles into a dimer and transports Zn2+," Journal of Neurochemistry, vol. 140, no. 2, pp. 280-293, 2017.

[19] R. C. Dunn, "Compact, inexpensive refractive index detection in femtoliter volumes using commercial optical pickup technology," Analytical Methods, vol. 11, no. 17, pp. 2303-2310, 2019.

[20] Y. Zhang, Y. Zhang, L. Zhu, P. He, and Q. Wang, "High sensitivity detection of Escherichia coli based on the measurement of $\beta$-galactosidase activity by microchip capillary electrophoresis combined with field-amplified sample injection," Analytical Methods, vol. 11, no. 11, pp. 1558-1565, 2019.

[21] P. S. Peixoto, I. V. Tóth, S. Machado et al., "Screening of sulfonamides in waters based on miniaturized solid phase extraction and microplate spectrophotometric detection," Analytical Methods, vol. 10, no. 7, pp. 690-696, 2018.

[22] R. Zhang, M. Zhang, Y. Zhang, H. Yan, X. Li, and W.-H. Xie, "The simultaneous detection of food dyes from different samples in a 96-well plate by spectrophotometry," Analytical Methods, vol. 11, no. 45, pp. 5793-5802, 2019.

[23] P. Atkins, L. Ernyei, W. Driscoll, R. Obenauf, and R. Thomas, "Spectroscopy," 2011.

[24] European Medicines Agency, Guideline on Bioanalytical Method Validation EMEA/CHMP/EWP/192217/2009, European Medicines Agency, Amsterdam, Netherlands, 2011.

[25] J. N. Miller and J. C. Miller, Statistics and Chemometrics for Analytical Chemistry, Pearson, Harlow, UK, 5th edition, 2005.

[26] B. J. R. Gregório, A. M. Pereira, S. R. Fernandes et al., "Flowbased dynamic approach to assess bioaccessible zinc in dry dog food samples," Molecules, vol. 25, no. 6, p. 1333, 2020. 